\title{
Substrates modified by advanced glycation end-products cause dysfunction and death in retinal pericytes by reducing survival signals mediated by platelet-derived growth factor
}

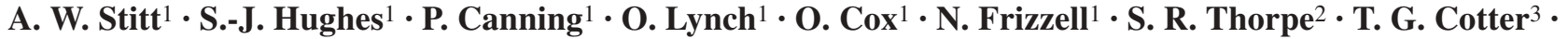 \\ T. M. Curtis ${ }^{1} \cdot$ T. A. Gardiner ${ }^{1}$ \\ ${ }^{1}$ Ophthalmic Research Centre, Ophthalmology \& Vision Science, Queen's University Belfast, Royal Victoria Hospital, Belfast, \\ Northern Ireland, UK \\ ${ }^{2}$ Department of Chemistry \& Biochemistry, University of South Carolina, Columbia, South Carolina, USA \\ ${ }_{3}^{3}$ Biochemistry Department, Bioscience Research Institute, University College Cork, Cork, Ireland
}

\begin{abstract}
Aims/hypothesis. Premature death of retinal pericytes is a pathophysiological hallmark of diabetic retinopathy. Among the mechanisms proposed for pericyte death is exposure to AGE, which accumulate during diabetes. The current study used an in vitro model, whereby retinal pericytes were exposed to AGE-modified substrate and the mechanisms underlying pericyte death explored.

Methods. Pericytes were isolated from bovine retinal capillaries and propagated on AGE-modified basement membrane (BM) extract or non-modified native BM. The extent of AGE modification was analysed. Proliferative responses of retinal pericytes propagated on AGE-modified BM were investigated using a 5-bromo-2-deoxy-uridine-based assay. The effect of extrinsically added platelet-derived growth factor (PDGF) isoforms on these proliferative responses was also analysed alongside mRNA expression of the PDGF receptors. Apoptotic death of retinal pericytes grown on AGE-modified BM was investigated using terminal deoxynucleotidyl transferase-mediated dUTP nick end-labelling labelling, mitochondrial membrane
\end{abstract}

depolarisation and by morphological assessment. We also measured both the ability of PDGF to reverse Akt dephosphorylation that was mediated by AGE-modified BM, and increased pericyte apoptosis.

Results. Retinal pericytes exposed to AGE-modified $\mathrm{BM}$ showed reduced proliferative responses in comparison to controls $(p<0.05-0.01)$, although this effect was reversed at low-AGE modifications. PDGF mRNA levels were differentially altered by exposure to low and high AGE levels, and AGE-modified BM caused significantly increased apoptosis in retinal pericytes. Pre-treatment of AGE-modified BM with PDGF-AA and $-\mathrm{BB}$ reversed the apoptosis $(p<0.05-0.001)$ and restored Akt phosphorylation in retinal pericytes.

Conclusions/interpretation. Evidence suggests that substrate-derived AGE such as those that occur during diabetes could have a major influence on retinal pericyte survival. During diabetic retinopathy, AGE modification of vascular BM may reduce bioavailability of pro-survival factors for retinal pericytes.

Keywords Advanced glycation - Apoptosis . Basement membrane - Diabetic retinopathy ·

Platelet-derived growth factor $\cdot$ Retinal pericytes
Received: 22 March 2004 / Accepted: 1 June 2004

Published online: 22 October 2004

(C) Springer-Verlag 2004

\section{A. W. Stitt $(\bullet)$}

Ophthalmic Research Centre, Ophthalmology \& Vision Science, Queen's University Belfast, Royal Victoria Hospital,

Grosvenor Road, Belfast, Northern Ireland, BT12 6BA, UK

E-mail: a.stitt@qub.ac.uk

Tel.: +44-2890-632546, Fax: +44-2890.263368

Abbreviations: BM, basement membrane .

BrdU, 5-bromo-2-deoxy-uridine - CEL, carboxyethyl lysine ·

CML, carboxymethyl lysine $\cdot$ HSP, heparan sulfate

\section{Introduction}

Retinal pericytes are intimately associated with the vascular endothelium and have a critical role in maintaining the functional integrity of the capillary unit

proteoglycans · PDGF, platelet-derived growth factor ·

PKB, protein kinase B - TUNEL, terminal deoxynucleotidyl transferase-mediated dUTP nick end-labelling

Conflict of interest: None of the authors have a conflict of interest. 
[1]. As contractile cells with well-developed actin microfilamentous networks, pericytes are thought to alter capillary luminal diameter and regulate retinal blood flow in response to a range of endotheliumderived vasomodulators [2, 3]. Retinal pericytes also appear to have a critical trophic function in promoting vessel maturation and endothelial survival via a complex molecular cross-talk between both retinal microvascular cell types [4].

During diabetes the retinal pericyte undergoes a range of pathophysiological responses, which may directly contribute to progressive retinal vasodegeneration. Hyperglycaemia-mediated pericyte dysfunction and, ultimately, premature pericyte death are a major pathogenic consequence of diabetic retinopathy [5], although the precise nature of the biochemical insult and of the subsequent death response remains illdefined. A range of in vitro and in vivo studies have demonstrated that retinal pericytes enter an apoptosisrelated death pathway after long-term diabetes in humans $[6,7]$, during experimental diabetes or hyperhexosaemia $[8,9]$, and after exposure to biochemical insults in culture $[10,11]$.

It has been proposed that hyperglycaemia contributes to diabetic microvasculopathy by promoting the formation of AGE [12, 13]. These adducts form through oxidation and rearrangement reactions of early non-enzymatic glycation products, which produce a class of irreversible moieties possessing distinctive chemical cross-linking and biological properties. More recently it has become clear that AGE can also form via the reaction of carbonyl intermediates [14]. Regardless of their chemical derivation, AGE modifications can have a significant impact on protein structure and function, often resulting in deleterious effects on cell responses and survival [12]. The precise role played by these adducts in the pathogenesis of diabetic retinopathy has not been fully defined, although experimental studies have demonstrated hat AGE may be responsible for retinal vascular cell death and closure, and that AGE-inhibiting compounds can prevent this pathology [12]. In diabetes, AGE have been localised to various tissues and organ systems, including the retinal microvasculature and neuroglia $[15,16,17]$. They also form on retinal vascular basement membranes (BM) during diabetes [15].

In anchorage-dependent cells such as retinal pericytes, apoptotic death can be initiated through blockage of important BM-mediated survival signals [18]. Under normal physiological conditions, cells receive survival stimuli whose effects are mediated by intracellular intermediates such as phosphatidylinositol3'kinase-mediated activation of the Ser/Thr kinase Akt/protein kinase $\mathrm{B}(\mathrm{PKB})$, which in turn inhibits pro-apoptotic effectors [19]. In vivo, AGE initiate inappropriate cross-linking of BM component proteins at an accelerated rate in diabetes, causing conformational change that affects heparin sulphate content, charge selectivity, configuration of receptor-recognition motifs, vascular permeability responses and vessel distendability [20].

Within retinal capillaries, pericytes are totally enveloped by BM and because they lie outside the blood retinal barrier, are reliant on trophic factors derived from endothelium and glia [21]. It could be reasonably assumed that enhanced AGE modification of BM components during diabetes restricts the bioavailability of heparin-binding growth and/or survival factors such as platelet-derived growth factor (PDGF). PDGF is a multifunctional dimeric peptide occurring as either $\mathrm{AA}, \mathrm{BB}$ homodimers or $\mathrm{AB}$ heterodimer isoforms. These dimeric configurations differentially activate the $a$ or $b$ subunits of the PDGF tyrosine kinase receptor and ligand binding studies have shown that the $\alpha$-subunit binds either the A or B chain, and the $\beta$-subunit binds only the $\mathrm{B}$ chain $[22,23]$. These ligand/receptor combinations account for a wide range of PDGF-mediated effects in a range of cell types. Indeed, PDGF is known to be a potent mitogen, have the ability to regulate extracellular matrix deposition, and act as a pro-survival factor in many tissues during development, responses to injury and in maintenance of normal cell function [24]. Effective removal of PDGF bio-activity can have profound pathologic effects in tissues [25, 26, 27].

In combination with the low replicative capacity of retinal pericytes both in health and during diabetes, AGE-mediated depletion of BM-immobilised growth factors has important survival implications for pericytes and the progression of diabetic retinopathy [25, $28,29]$. The current study adopted an in vitro model by exposing retinal pericytes to a well-characterised and defined AGE-modified substrate and investigating pericyte replicative capacity and apoptotic death. The potential for PDGF to modulate these responses was also determined.

\section{Materials and methods}

\section{Cell isolation}

Bovine eyes were obtained from a local abattoir and retinal microvascular pericytes isolated by selective sieving and digestion as previously described [30]. The cells were propagated on T25 Falcon flasks in DMEM (Gibco, Paisley, UK) containing $5 \mathrm{mmol} / \mathrm{l}$ glucose and 20\% FCS (Gibco).

\section{AGE modification of cell substrate}

Upon establishment, retinal pericytes were propagated on growth-factor-depleted basement membrane matrix (Native BM), extracted from Engelbreth-Holm-Swarm mouse sarcoma (Matrigel, BD Biosciences, Oxford, UK). AGE-modified substrate (AGE-modified BM) was formed by incubating Matrigel in $0.1-100 \mathrm{mmol} / \mathrm{l}$ glycoaldehyde $[31,32]$. To ensure that observed effects were due to AGE-modified BM alone, the substrate was pre-modified and the active glycating agent careful- 
ly washed away using PBS. Any free aldehyde groups remaining were "neutralised" by incubating with $50 \mathrm{mmol} / \mathrm{l}$ sodium borohydride $\left(50 \mathrm{mmol} / \mathrm{l}\right.$ overnight at $\left.4{ }^{\circ} \mathrm{C}\right)$. The substrate was further washed prior to seeding cells. Control matrices were treated in a similar way without the addition of glycoaldehyde for 4 hours at $37^{\circ} \mathrm{C}$.

The extent of carboxymethyl lysine (CML) and carboxyethyl lysine (CEL) formation was quantified by assessment of lysine modification using gas chromatography/mass spectrometry as previously described [33]. For AGE fluorescence measurements, control and AGE-BM were analysed for Maillard type fluorescence (excitation $370 \mathrm{~nm}$, emission $440 \mathrm{~nm}$ ) on a spectrofluorimeter (Tecan Safire, Reading, UK). The results were normalised to the protein content of the solution.

The extent of cross-linking in AGE-BM was determined from the kinetics of the proteolytic digestion of collagen by pepsin. The control and AGE-modified substrates were suspended in $0.5 \mathrm{~mol} / \mathrm{l}$ acetic acid and digestion was initiated by adding fresh pepsin (Sigma Chemical, Poole, UK) at a concentration of $50 \mu \mathrm{g}$ pepsin/mg collagen. The plates containing the substrates were sealed to prevent evaporation and the samples were then incubated for up to 24 hours at $37{ }^{\circ} \mathrm{C}$ in a hot air oven with mild agitation. We removed $500-\mu$ l aliquots of the supernatant at 2-hourly intervals and mixed them with an equal volume of concentrated hydrochloric acid. The samples were then hydrolysed in a vacuum at $110{ }^{\circ} \mathrm{C}$ for 24 hours. After hydrolysis the samples were dried in a speed-vac (Howe, Banbury, UK) prior to analysis of free hydroxyproline.

The levels of the collagen-specific imino acid hydroxyproline were detected in the hydrolysed protein samples using the Stegemann and Stalder method [34], in which the protein samples that had been digested by pepsin were hydrolysed to release free hydroxyproline The absorbance of the samples was read at $570 \mathrm{~nm}$ (Tecan Safire spectrophotometer) and the free hydroxyproline in each sample was calculated according to the values determined for a standard curve of 1-hydroxyproline.

\section{Supplementation of substrates with PDGF isoforms}

To determine the modulatory role of matrix-immobilised growth factors in pericyte dysfunction and death, PDGF isoforms (in the form of AA or BB homodimers at $50 \mathrm{ng} / \mathrm{ml}$ ) (Sigma) were placed on top of both native BM and AGE-modified BM $(15 \mathrm{ng} / \mathrm{ml})$ and incubated for 3 to $6 \mathrm{~h}$ at $4{ }^{\circ} \mathrm{C}$. Excess PDGF solution was removed and the matrices washed once in basal growth medium prior to seeding cells for the various assays.

\section{PDGF receptor expression}

Retinal pericyte RNA was extracted using the RNeasy Mini Kit (Qiagen, Crawley, UK). The quantity of RNA in each experimental group was determined spectrophotometrically (U 1100 model, Hitachi, Berkshire, UK) and the purity and quality of each RNA sample was estimated by visualisation of clear $18 \mathrm{~S}$ and $28 \mathrm{~S}$ ribosomal RNA bands after electrophoresis of $1 \mu \mathrm{g}$ of each sample on a $1 \%$ agarose gel.

The RNA from each extraction was reverse transcribed into cDNA using a 1st Strand cDNA Synthesis Kit (Life Technologies, Paisley, UK) and random hexamer primers (Boehringer Mannheim, Mannheim, Germany). Real-time PCR was conducted for quantitative analysis of mRNA expression using sequence-specific primers for PDGF- $\alpha$ (forward: 5' CAA ACC CTG AGA CCA CAA TG 3', reverse: 5' TCC CCC AAC AGT AAT CCA AG 3', -235-bp fragment) and $P D G F-\beta$ (forward: 5' TGC CTC AGC CAA ATG TCA CC 3', reverse:
5' TGC TCA CCA CCT CGT ATT CC 3', 159-bp fragment). Primers to amplify the housekeeping gene acidic ribosomal phosphoprotein were also designed: (forward: 5' CGA CCT GGA AGT CCA ACT AC 3', reverse: 5' ATC TGC TGC ATC TGC TTG 3', 109-bp fragment).

Real-time PCR was performed using a LightCycler rapid thermal cycler system (Roche, Hertfordshire, UK) according to protocols outlined previously [35]. The PCR reaction was performed in glass capillary reaction vessels (Roche) of $20 \mu \mathrm{l}$ volume with $0.5 \mu \mathrm{mol} / 1$ primers. Reaction buffer, $2.5 \mathrm{mmol} / \mathrm{l}$ $\mathrm{MgCl}_{2}$, dNTPs, Hotstart Taq DNA polymerase and SYBR Green I were included in the QuantitTect LightCycler-SYBR Green PCR Master Mix (Qiagen). Amplification of cDNAs involved a 15-minute denaturation step followed by 40 cycles with denaturation at $95{ }^{\circ} \mathrm{C}$ for $15 \mathrm{~s}, 55-58{ }^{\circ} \mathrm{C}$ annealing for $20 \mathrm{~s}$ and $72{ }^{\circ} \mathrm{C}$ for an appropriate extension time (5-25 s). Fluorescence from SYBR Green I bound to the PCR product was detected at the end of each $72{ }^{\circ} \mathrm{C}$ extension period. The specificity of the amplification reactions was confirmed by melting curve analysis and subsequently by agarose gel electrophoresis [35]. The quantification data were analysed with the LightCycler analysis software as described previously [35]. The baseline of each reaction was equalised by calculating the mean value of the five lowest measured data points for each sample and subtracting this from each reading point. Background fluorescence was removed by setting a noise band. The number of cycles at which the best-fit line through the loglinear portion of each amplification curve intersects the noise band is inversely proportional to the log of copy number. A dilution series of a reference cDNA sample was used to generate a standard curve, against which the experimental samples were quantified. For each gene, PCR amplifications were performed in triplicate on at least two independent RT reactions.

\section{Pericyte replication}

Pericyte DNA replication was examined on native BM, AGE$\mathrm{BM}$ and $\mathrm{BM}$ supplemented with PDGF-AA or PDGF-BB using an assay in which 5-bromo-2-deoxy-uridine (BrdU) incorporation into DNA is quantified. Pericytes were seeded onto 96-well microtitre plates coated with either native $\mathrm{BM}$ or AGE-modified $\mathrm{BM}$ at population densities of $3 \times 10^{5}$ cells per well (six wells per experimental condition). Cells were grown on AGE-modified BM of various degrees of modification and for increasing time periods (12-48 h). Native BM was used as a control substrate. Likewise, pericyte replication was determined after supplementation of the substrates with PDGF-(AA or $\mathrm{BB})$. BrdU incorporation was measured using a commercially available kit (Roche) and was carried out according to the manufacturer's instructions. Briefly, BrdU was added to each well and incubated overnight at $37^{\circ} \mathrm{C}$. The medium was removed and cells were washed with PBS supplemented with $10 \% \mathrm{FCS}$ and fixed with ethanol $(70 \%)$ in $\mathrm{HCl}$ (final concentration $0.5 \mathrm{~mol} / \mathrm{l})$, the latter being done for 30 minutes at $-20{ }^{\circ} \mathrm{C}$. Following fixation, the cells were washed and cellular DNA was partially digested by incubation with nuclease solution for 30 minutes in the absence of $\mathrm{CO}_{2}$. The cells were then washed and incubated with anti-BrdU peroxidase conjugate antibody for 30 minutes at $37{ }^{\circ} \mathrm{C}$. Finally the cells were washed and subsequently incubated with the peroxidase substrate solution, which catalyses the cleavage of the soluble chromogenic ABTS substrate and yields a coloured reaction product. BrdU incorporation was quantified by measurement of optical density at $405 \mathrm{~nm}$ using a reference filter of $490 \mathrm{~nm}$ (SLT rainbow advanced scanning microplate photometer, with Biolise software, Tecan Instruments, Groedig, Austria). 
Pericyte apoptosis

In situ apoptotic DNA fragmentation demonstrated by TUNEL. Fragmented DNA within apoptotic cells was stained in situ by the terminal deoxynucleotidyl transferase-mediated dUTP nick end-labelling (TUNEL) method. The pericytes used for TUNEL were grown on chamber slides pre-coated with native BM or BM modified with glycoaldehyde (as outlined above) for 24 hours. The experimental procedure was followed in accordance with the manufacture's instructions (Promega, Southampton, UK). Briefly, pericytes to be stained by TUNEL were fixed for 20 minutes in 4\% PFA, washed in PBS and permeabilised using $0.2 \%$ Triton $\mathrm{X}-100$. The cells were then covered in equilibration buffer for 10 minutes, after which they were subjected to the incubation buffer containing the fluorescein-labelled dUTP and terminal deoxynucleotide transferase enzyme. After this step, we avoided exposure of the cells to light. Following 1 hour of incubation at $37^{\circ} \mathrm{C}$, the incorporation of dUTP into the nicked DNA was terminated. The cells were then washed in PBS and their nuclei counterstained with propidium iodide (Molecular Probes, Eugene, Ore., USA). The slides were mounted in Vectashield (VectorLabs, Peterborough, UK). DNAase treatment of normal pericytes provided positive controls, while deoxynucleotide transferase enzyme omission was used as a negative control.

Pericyte mitochondrial depolarisation. The cationic fluorescent probe JC-1 (Molecular Probes) was used to detect mitochondrial depolarisation, an early event in the apoptotic cell death response. The JC-1 probe acts in a potential-dependent manner, accumulating in mitochondria in healthy cells and resulting in a shift of fluorescence emission from green to red [36]. During apoptosis, mitochondria are incapable of concentrating the fluorophore, therefore this shift of fluorescence emission does not occur, leading to a net decrease in the red : green fluorescence intensity ratio.

Retinal pericytes (passage 3-4) were propagated on either AGE-modified BM or native BM in T25 flasks. After $24 \mathrm{~h}$, JC-1 dye was added to the culture medium for $30 \mathrm{~min}$ (final concentration $1 \mu \mathrm{g} / \mathrm{ml}$ ). Cells were harvested by gently scraping with a rubber policeman, pelleted, re-suspended in PBS and analysed immediately using a FACSCaliber flow cytometer (Becton Dickinson, Oxford, UK). We collected 50,000 events per sample and recorded the red (J-aggregates) and green (JC-1) fluorescence intensities on the FL-1 and FL-2 channels respectively. All samples were analysed in triplicate.

Morphological assay. For use in a cell death morphological assay pericytes at passage 2-4 were seeded on 3-cm diameter Petri dishes (Falcon BD Biosciences, Oxford, UK) that had been coated in AGE-modified BM or native BM control substrates. The cells were analysed after 24 hours whilst still preconfluent (cell density in the range of 50-80 cells per low magnification field). This density ensured minimal overlap with adequate separation of individual cells for accurate counting after treatment. For analysis, the cultures were fixed with $4 \%$ paraformaldehyde in $0.1 \mathrm{~mol} / \mathrm{l}$ phosphate buffer at $\mathrm{pH} 7.4$. In initial experiments 20 fields were counted and a running mean was plotted to determine the minimum number of fields that would accurately reflect the population mean. It was found that no more than six fields were ever necessary to calculate the proportion of normal, apoptotic and necrotic cells in any given culture. For each individual culture dish, therefore, six fields approximately equidistant both from each other and the edge of the dish were counted in a clockwise fashion. In practice this process yielded a sample of between 100 and 180 cells per culture. Apoptotic pericytes were typified by gross cyto- plasmic shrinkage, retraction from contiguous cells and rounding-up with a phase-bright halo.

Retinal pericytes undergoing apoptotic death (as depicted by phase microscopy above) were prepared for transmission electron microscopy by detaching cells with trypsin/versene prior to pelleting. The cell pellets were then fixed with $2.5 \%$ glutaraldehyde in $0.1 \mathrm{~mol} / 1$ sodium cacodylate buffer $(\mathrm{pH} 7.2)$ containing $10 \mathrm{mmol} / 1 \mathrm{MgCl}_{2}$. The fixed cell pellets were osmicated, dehydrated, and embedded in epoxy resin for ultrathin sectioning.

SDS-PAGE and western blotting. Since pro-survival pathways of PDGF-receptor signalling results in up-regulation of antiapoptotic proteins that is mediated by phosphorylation of Akt, we sought to evaluate this pathway after exposure to AGEmodified BM with and without PDGF supplementation. Protein samples of pericytes that were grown on variously modified AGE-modified BM or native BM controls for 24 hours were prepared by scraping the cells off the flasks in an extraction buffer solution of PBS containing protease inhibitors (Complete Mini, Boehringer Mannheim). The samples were solubilised and the protein concentration was determined using the BCA protein kit (Pierce, Rockford, Ill., USA). SDS-PAGE was conducted according to standard protocols with protein samples $(10 \mu \mathrm{g})$ diluted in Laemmli lysis buffer (Bio-Rad, Richmond, Calif., USA) containing $2 \% \quad \beta$-mercaptoethanol. Equal amounts of each protein sample were separated on denaturing 4-20\% SDS gels (Bio-Rad) and transferred onto nitrocellulose membrane (Bio-Rad). After blocking, the membrane was probed with a polyclonal antibody to phosphorylated (ser 473)-Akt (phospho-Akt) (Cell Signaling Technology, Beverley, Mass., USA ) for 1 hour. The membrane was then washed and incubated in a goat anti-rabbit secondary antibody with a horseradish peroxidase-conjugate (Dako, Ely, UK) for 2 hours. After washing, immunoreactivity was detected using enhanced chemiluminescence (Amersham, Little Chalfont, UK) and exposure to Hyperfilm X-ray film (Amersham).

Statistical analysis. Statistical analysis of all data was performed using one-way ANOVA and a Tukey-Kramer multiple comparisons test. Data were considered significant at a $p$ value of less than 0.05 and a CI of $95 \%$.

\section{Results}

Substrate AGE modification. Glycoaldehyde-modification of Matrigel for 4 hours resulted primarily in the formation of CML, with only a modest amount of CEL formed at $500 \mathrm{mmol} / \mathrm{l}$ glycoaldehyde (Table 1). Prolonged incubation times produced increasing levels of CEL and CML, although CEL production was minor compared to CML (Table 1). In addition, the AGE-related autofluorescence properties of these matrices showed increasing levels of fluorescence at $370 / 440 \mathrm{~nm}$, which was dependent on glycoaldehyde concentration (Fig. 1). The release of hydroxyproline, which was used as an indicator of cross-linking, was significantly decreased in the AGE-modified BM samples, both in terms of total hydroxyproline content (data not shown) and time taken for $50 \%$ digestion (Table 2). In particular, the $100 \mathrm{mmol} / \mathrm{l}$ and $500 \mathrm{mmol} / \mathrm{l}$ samples showed a significant increase in protein cross-linking (Table 2$)(p<0.03, n=3)$. 


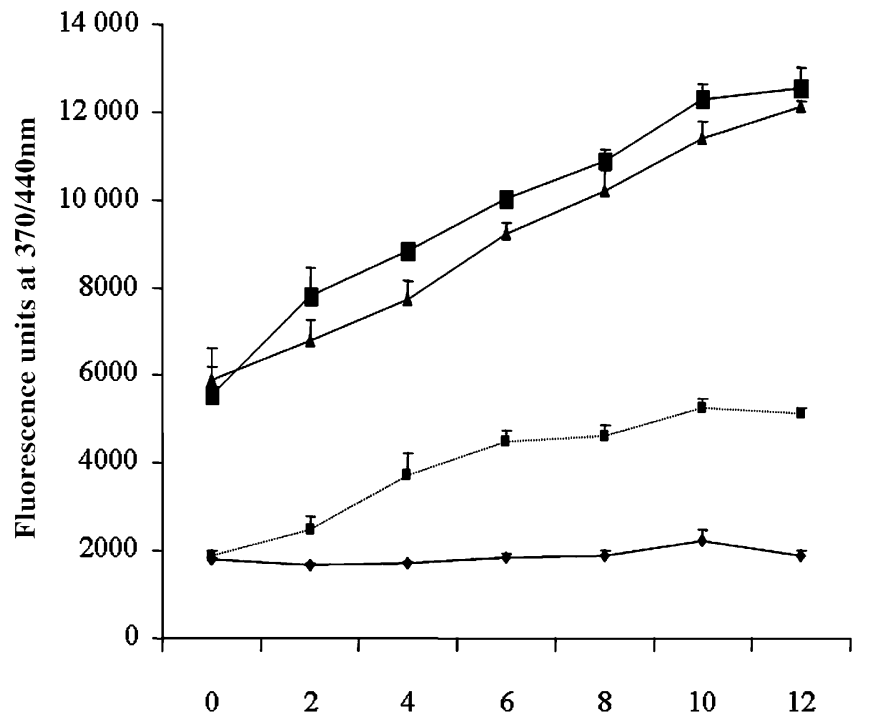

Time, hours

Fig. 1. AGE fluorescence of proteolytically digested AGEmodified matrices. Glycoaldehyde incubation with basement membrane extract produces a significant concentration and time-dependent increase in AGE fluorescence. Diamond: $0 \mathrm{mmol} / \mathrm{l}$ glycoaldehyde; small square: $10 \mathrm{mmol} / \mathrm{l}$ glycoaldehyde; triangle: $100 \mathrm{mmol} / \mathrm{l}$ glycoaldehyde; large square: $500 \mathrm{mmol} / \mathrm{l}$ glycoaldehyde. Error bars represent $\mathrm{SD} \pm$ mean

Table 1. Carboxymethyl lysine (CML) and carboxyethyl lysine (CEL) content in AGE-modified matrices

\begin{tabular}{lll}
\hline Sample & $\begin{array}{l}\text { CML, } \\
\text { mmol/mol lysine }\end{array}$ & $\begin{array}{l}\text { CEL, } \\
\text { mmol/mol lysine }\end{array}$ \\
\hline Control BM & 0.62 & 0 \\
$4 \mathrm{~h} 10 \mathrm{mmol} / 1$ & 9.16 & 0 \\
$4 \mathrm{~h} 100 \mathrm{mmol} / 1$ & 7.83 & 0 \\
$4 \mathrm{~h} 500 \mathrm{mmol} / \mathrm{l}$ & 14.54 & 0.613 \\
24 h $10 \mathrm{mmol} / 1$ & 9.35 & 0.106 \\
$24 \mathrm{~h} 100 \mathrm{mmol} / 1$ & 21.84 & 0.818 \\
$24 \mathrm{~h} 500 \mathrm{mmol} / 1$ & 52.29 & 1.059 \\
\hline
\end{tabular}

GCMS analysis of native BM (Matrigel) and BM modified with 10,100 or $500 \mathrm{mmol} / \mathrm{l}$ glycoaldehyde for 4 or 24 hours. Marked formation of CML occurred in all glycoaldehyde-treated BM, but CEL only appears to form after long exposure times and/or with high concentrations of glycoaldehyde

Table 2. Cross-linking of AGE-modified matrices

\begin{tabular}{ll}
\hline Sample & $\begin{array}{l}\text { Time for } 50 \% \\
\text { digestibility } \\
\text { (minutes) }\end{array}$ \\
\hline Control BM (0 mmol/l) & 248.87 \\
AGE-modified BM $(10 \mathrm{mmol} / \mathrm{l})$ & 309.01 \\
AGE-modified BM $(100 \mathrm{mmol} / \mathrm{l})$ & $582.82^{*}$ \\
AGE-modified BM $(500 \mathrm{mmol} / \mathrm{l})$ & $598.18^{*}$ \\
\hline
\end{tabular}

Glycoaldehyde cross-linking results in an increased time for proteolytic digestion. Results are expressed as time taken for $50 \%$ of the protein to be digested. * Significantly different from control $(p<0.03)$
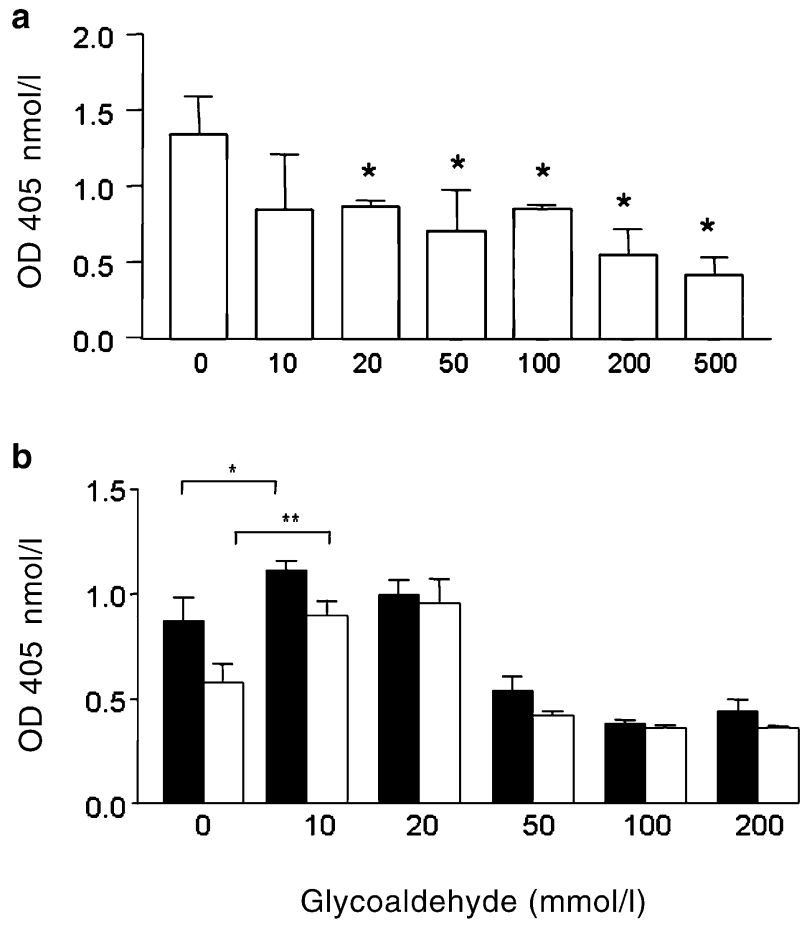

Fig. 2. Proliferation of retinal pericytes on AGE-modified matrices. BrdU incorporation into replicating DNA in pericytes grown on native BM and AGE-modified BM modified with increasing concentrations of glycoaldehyde. After 12 hours (a) there was a significant reduction in pericyte proliferation compared to native BM (represented as $0 \mathrm{mmol} / \mathrm{l}$ glycoaldehyde). Similar patterns were evident for 24 and 48 hours (data not shown). PDGF supplementation of the AGE-modified matrices (b) alters the pericyte proliferative response. Black bars: effect of PDGF-AA (50 ng/ml); open bars: effect of PDGF-BB $(50 \mathrm{ng} / \mathrm{ml})$. The effects were quite similar and show that the AGE-mediated effect was reversed at low levels of modification. Error bars represent SD \pm mean. OD, optical density. $* p<0.05 ; * * p<0.01$

Pericyte proliferation on AGE-modified substrate. Retinal pericytes propagated on an AGE-modified $\mathrm{BM}$ demonstrated a significant reduction in BrdU incorporation into DNA when compared with native BM controls $(p<0.05-0.01)$. This response was dependent on the degree of AGE modification (Fig. 2a) and was similar for cells growing for 12, 24 or 48 hours on these substrates (data not shown). When native or AGE-modified BM was supplemented with PDGFAA or PDGF-BB (15 ng/ml) prior to seeding, the retinal pericytes demonstrated a significantly greater $(p<0.05-0.01)$ proliferative response than native BM controls, but only at low AGE modification levels (10-20 mmol/l glycoaldehyde) (Fig. 2b). PDGF supplementation at high-level AGE modifications (>50 mmol/l glycoaldehyde) did not enhance DNA replication. This was true for both PDGF-AA and PDGF-BB homodimers (Fig. 2b).

PDGF-receptor expression. Quantitiative real-time PCR demonstrated that PDGF-beta and PDGF-alpha 

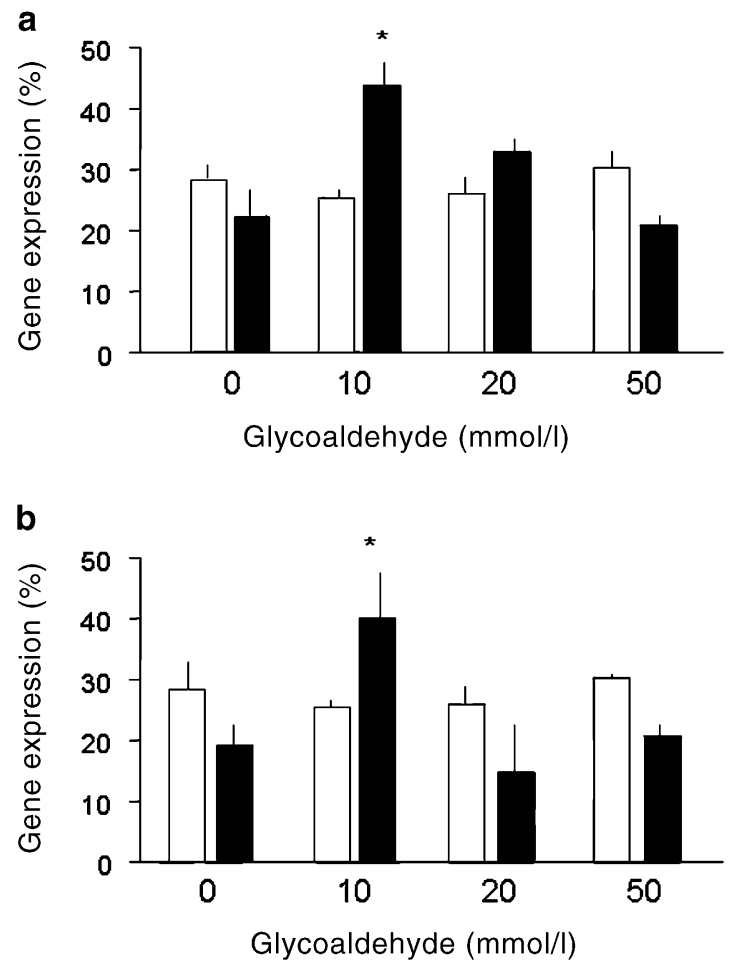

Fig. 3. PDGF-receptor expression by retinal pericytes on AGE-modified matrices. Real-time RT-PCR was conducted to determine changes in mRNA of $P D G F-\alpha(\mathbf{a})$ and $P D G F-\beta$ (b) (both represented by black bars) after pericytes were propagated on native $\mathrm{BM}(0 \mathrm{mmol} / \mathrm{l}$ glucoaldehyde $)$ or increasingly AGE-modified BM. Open bars: 28S housekeeping gene expression, mRNA levels of which did not alter between treatments. For both PDGF receptors mRNA levels increased at low levels of AGE modification $\left({ }^{*} p<0.05\right)$. More extensively cross-linked substrates failed to show any significant differences from controls. Error bars represent $\mathrm{SD} \pm$ mean

receptor mRNA expression levels were relatively low on native BM (Fig. 3a, b). However, exposure to AGE-modified BM caused a marked increase in mRNA expression for both these PDGF isoforms at low AGE modification levels $(p<0.05)$, and there was a similar response in PDGF-alpha and PDGF-beta receptor mRNAs (Fig. 3a and b respectively).

AGE-mediated apoptosis in pericytes. Fragmentation of pericyte DNA was investigated using the TUNEL assay and this demonstrated an AGE-mediated increase in TUNEL-positive nuclei (Fig. 4a-c). Cells grown on AGE-modified BM consistently demonstrated super-condensed chromatin, which was identified with propidium iodide. Many of these fragments proved to be TUNEL-positive also (Fig. $4 \mathrm{a}-\mathrm{c}$ ). Quantification of TUNEL-positive nuclei revealed a significant increase when retinal pericytes were propagated on AGE-modified BM $(p<0.01)$.

Mitochondrial depolarisation is a characteristic of early apoptotic change and the cationic fluorescent probe JC-1 was used in combination with flow cytom-
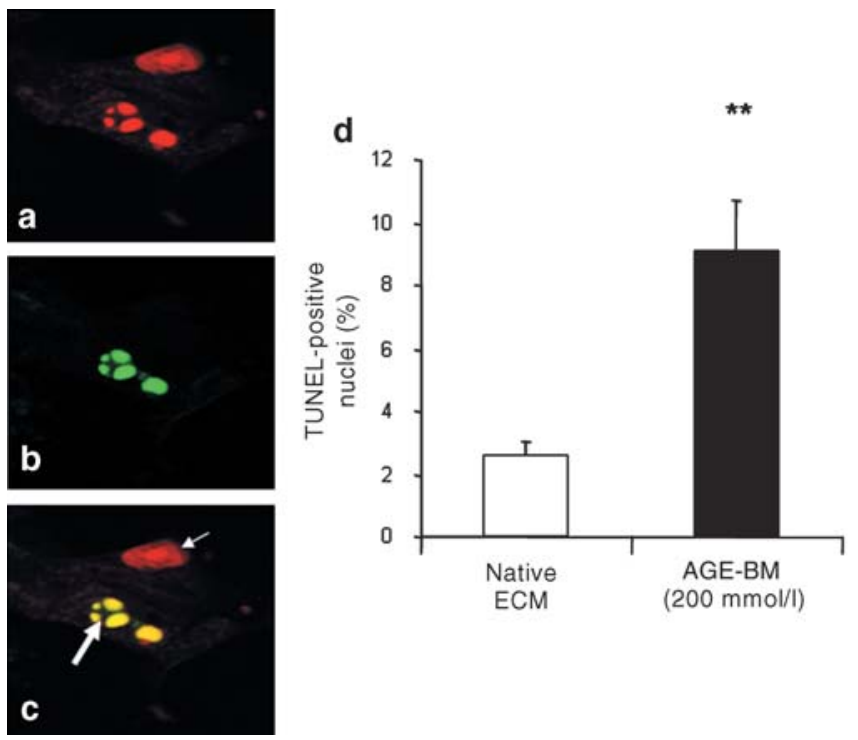

$(200 \mathrm{mmol} / \mathrm{l})$

Fig. 4. TUNEL labelling of retinal pericytes. TUNEL assay confirmed intranucleosomal DNA fragmentation in retinal pericytes grown on AGE-modified BM when compared to controls. Using confocal microscopy, propidium iodide stained all DNA red (a), while TUNEL-positive nuclei are represented by green immunofluorescence (b). Merged images (c) show some nuclei were also TUNEL-positive (large arrow), while others were negative (small arrow). Three separate experiments were conducted and TUNEL-positive nuclei were quantified on AGE-modified BM (200 mmol/l GA) (d). Exposure to AGEmodified BM caused a significant increase in intranucleosomal DNA fragmentation in retinal pericytes. Error bars represent $\mathrm{SD} \pm$ mean. ${ }^{* *} p<0.01$

etry to quantify this phenomenon in retinal pericytes. Cells grown on AGE-modified BM demonstrated a significant reduction in the red : green fluorescence ratio when compared to cells grown on unmodified Matrigel $(p<0.002)$ (Fig. 5a). Visualisation of this probe in pericytes using confocal microscopy showed that the mitochondria of pericytes grown on native Matrigel were polarised and therefore exhibited characteristic punctuated orange-red fluorescent staining of JC-1 aggregates (Fig. 5b, i-iii). When grown on AGE-modified BM, pericyte mitochondria were depolarised and exhibited a shift in fluorescence of the JC-1 monomer from red to green (Fig. 5b, iv-vi).

Pericytes grown on AGE-modified BM for longer periods ( $>4$ days) demonstrated distinct morphological changes when compared to native BM controls (Fig. 6). As determined by phase-contrast microscopy, there were typical morphological features of apoptosis, including condensation and shrinkage of the cell cytoplasm, a process which in the early stages resulted in a dendritic appearance (Fig. 6b). At a later stage, the apoptotic pericytes demonstrated the distinctive process of membrane blebbing, followed by cleavage of the blebs to yield apoptotic bodies (Fig. 6c). Although the blebbing and cytoplasmic cleavage were often observed in situ, some cells were seen to "round up" and 
a
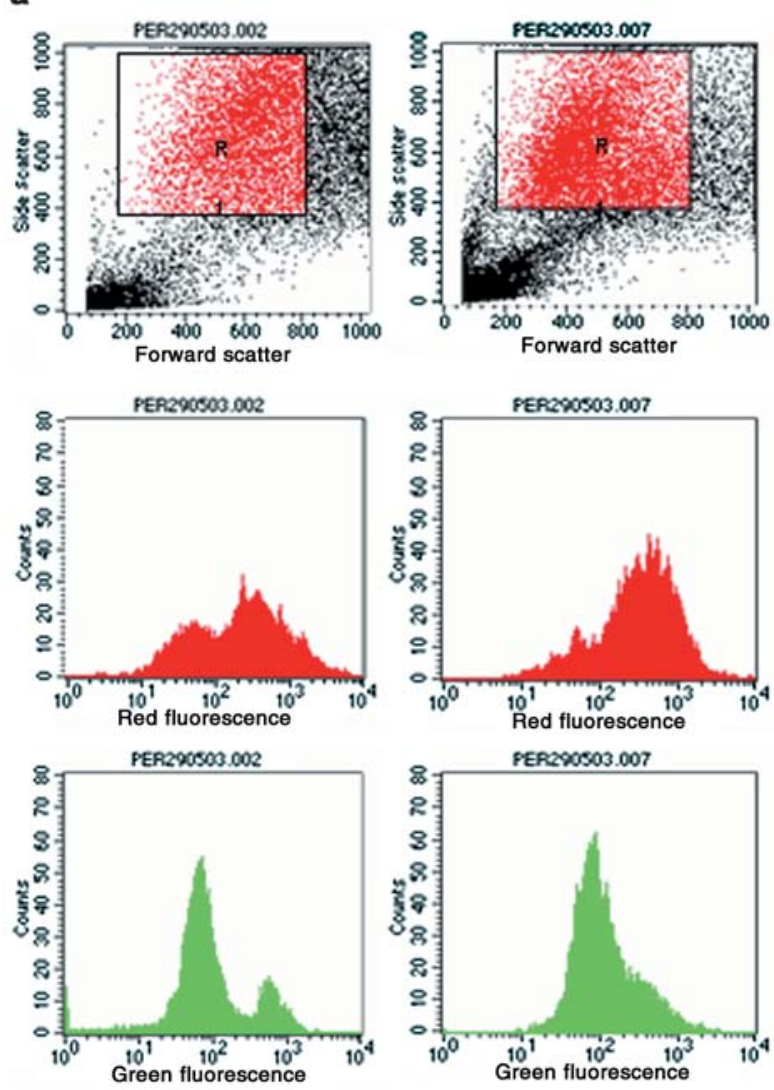
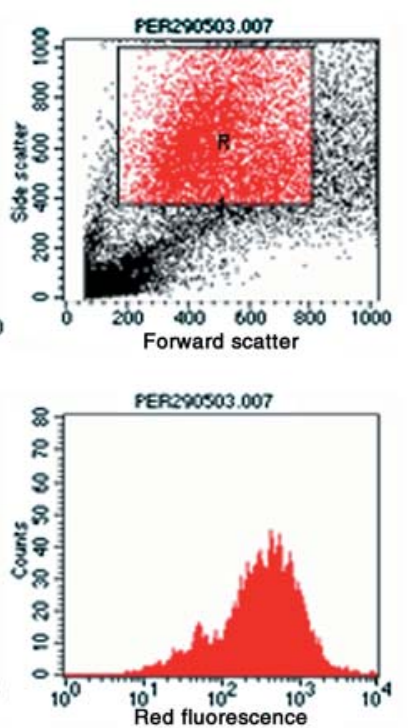

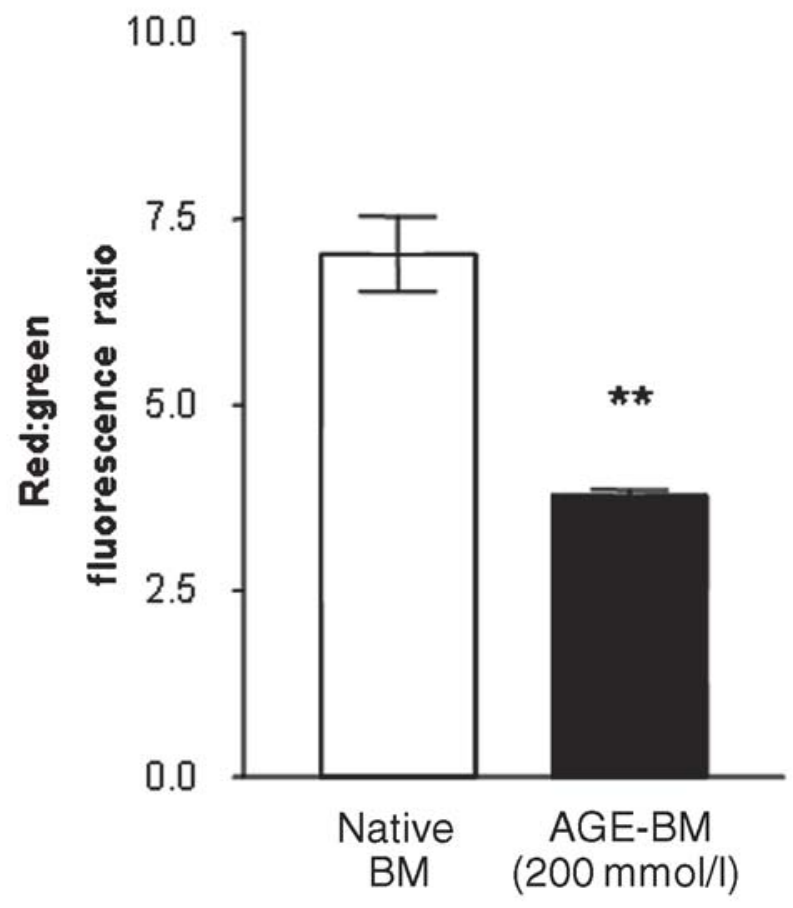

b

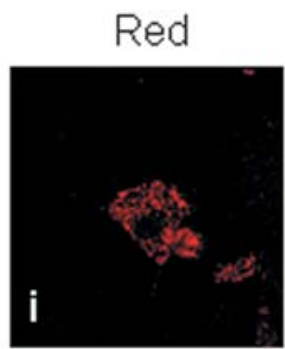

Green
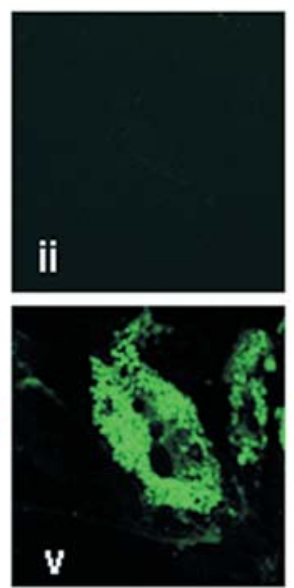

Merged
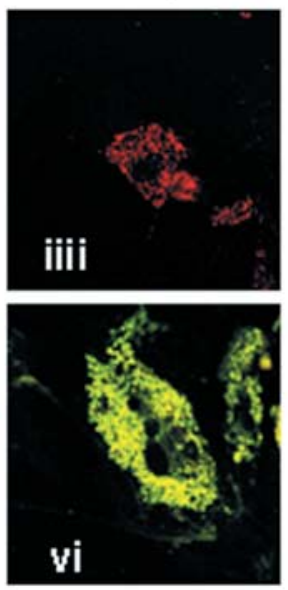

Fig. 5. AGE exposure causes hyperpermeability of pericyte mitochondria. a. Representative flow cytograms (left) of JC-1-stained retinal pericytes. The left-hand panels are of retinal pericytes grown on native BM for $24 \mathrm{~h}$, the right-hand panels are of retinal pericytes grown on AGE-modified BM ( $200 \mathrm{mmol} / \mathrm{l}$ glycoaldehyde) for $24 \mathrm{~h}$. The apoptotic cell population was gated on the basis of forward and side scatter and the red and green fluorescence profiles of this population monitored. The graph shows a significant reduction in the red : green fluorescence ratio in the cells grown on AGE-modified $\mathrm{BM}$ when compared to cells grown on native BM, indica- tive of increased apoptosis. The error bars represent SD \pm mean. $* * p<0.002$. b. Representative confocal microscopy images of JC-1-stained retinal pericytes grown on native BM (upper row) and AGE-modified BM (lower row). The mitochondria of pericytes grown on native Matrigel are polarised and therefore exhibit characteristic punctuated orange-red fluorescent staining (i-iii) of JC-1 aggregates. When grown on AGE-modified BM, pericyte mitochondria are depolarised and exhibit diffuse green JC-1 monomer fluorescence (iv-vi). This depolarisation event is a characteristic hallmark of early apoptosis 

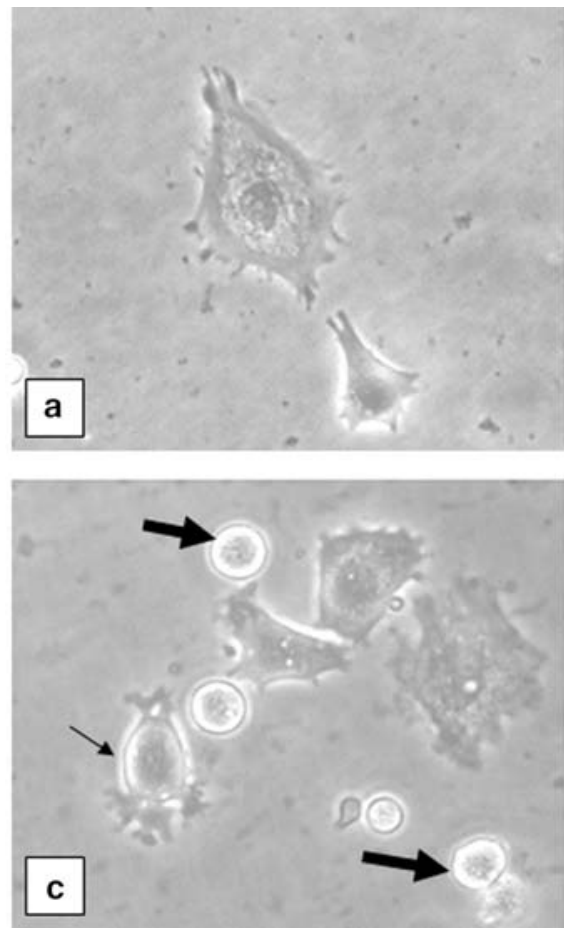

Fig. 6. Phase-contrast microscopy of pericyte apoptosis, with micrographs of retinal pericytes and changes in appearance on exposure to AGE-modified BM. Pericytes on native BM showed a typical stellar shape with cell processes spread onto the substrate (a). Cells grown on AGE-modified BM for 24 hours generally showed a less flattened, spread-out shape, often with a phase-bright halo, indicating that they are risingup from their substrate (arrow) (b). More prolonged exposure to AGE-modified BM (4 days) indicated large numbers of pericytes that had cytoplasmic blebbing and were drawing processes away from the substrate (small arrow) (c). Other cells were completely rounded up with few if any cytoplasmic processes and appeared as phase-bright cells (large arrows) (c). Transmission electron microscopy of rounded-up pericytes growing on AGE-modified BM (d) demonstrated typical apoptosis characteristics such as nucelosomal cleavge and fragmentation of chromatin and vacuolated cytoplasm (indicated by C)

undergo detachment prior to cleavage. When cell shrinkage became evident, apoptotic retinal pericytes tended to have a phase-bright halo (Fig. 6b, c), this being particularly pronounced around blebs, apoptotic bodies and whole rounded-up cells immediately before detachment. Phase-bright (apoptotic) pericytes were readily distinguished from occasional necrotic cells, which remained attached to the substrate until at a relatively advanced stage in the process and, unlike those in apoptosis, tended to maintain their original profile with no evidence of condensation or shrinkage.

Transmission electron microscopy of rounded-up retinal pericytes in vitro revealed apoptotic characteristics as demonstrated by intranucleosomal cleavage resulting in chromatin clumping into distinct bodies contained within the confines of intact plasma membrane (Fig. 6d).
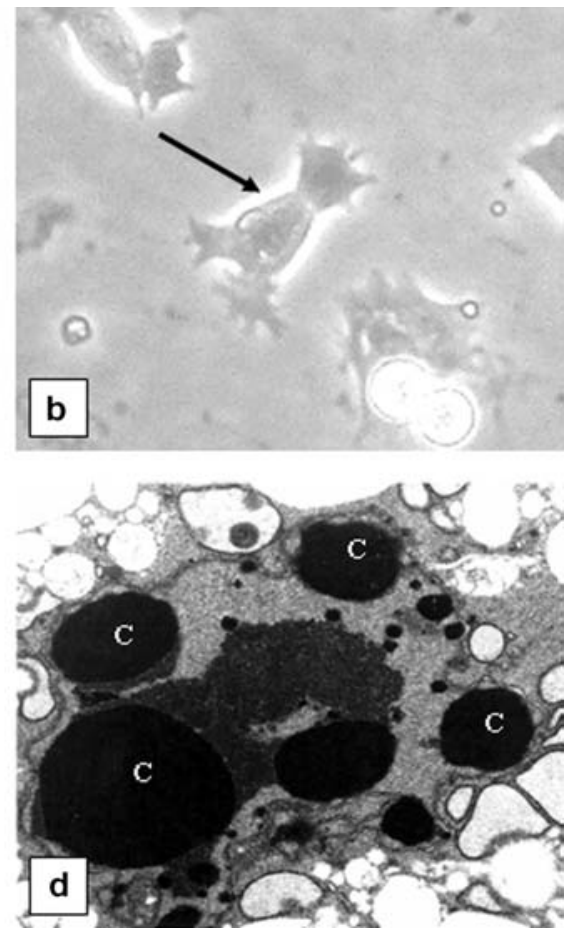

PDGF-mediated survival of AGE-exposed pericytes. Pericyte apoptotic responses were quantified and it was apparent that increasingly modified AGE-modified BM induced a significant step-wise increase in cell death when compared to native BM controls $(p<0.05)$. When AGE-modified BM was pre-treated with PDGF-AA or PDGF-BB there was a marked prevention of pericyte apoptotic death (Fig. 7a and b respectively). At low levels of AGE modification (10 mmol/l glycoaldehyde), PDGF-BB appeared more effective than PDGF-AA $(p<0.001)$, although at higher AGE levels both growth factors were equally effective at preventing pericyte death (Fig. 7).

Western blot analysis of phospho-Akt. Phosphorylation of the phosphatidylinositol-3-kinase-Akt/PKB as determined by western blot analysis, was down-regulated in pericytes grown on AGE-modified BM when compared to native BM controls (Fig. 8). However, when pericytes were exposed to AGE-modified BM that had been previously supplemented with PDGF$\mathrm{BB}$ there was no apparent reduction in phosphorylation of Akt.

\section{Discussion}

Previous studies in long-term diabetic rodents have demonstrated AGE accumulation in vascular BMs of retinal capillaries [15, 37, 38, 39]. This AGE accumulation may play a significant role in progression of diabetic retinopathy. Data from the current investigation suggest that BM accumulation of AGE can induce retinal pericyte dysfunction and pericyte death. Using a range of biochemical analyses, we character- 
ised the nature of the AGE-modified BM substrate used. It would have been ideal to compare the substrates prepared in vitro with retinal vascular BM extracts, but this was not possible. Nevertheless, the nature of the AGE formed and the extent of crosslinking in this in vitro system shares many protein characteristics with the extracellular matrix from diabetic animal models [40].
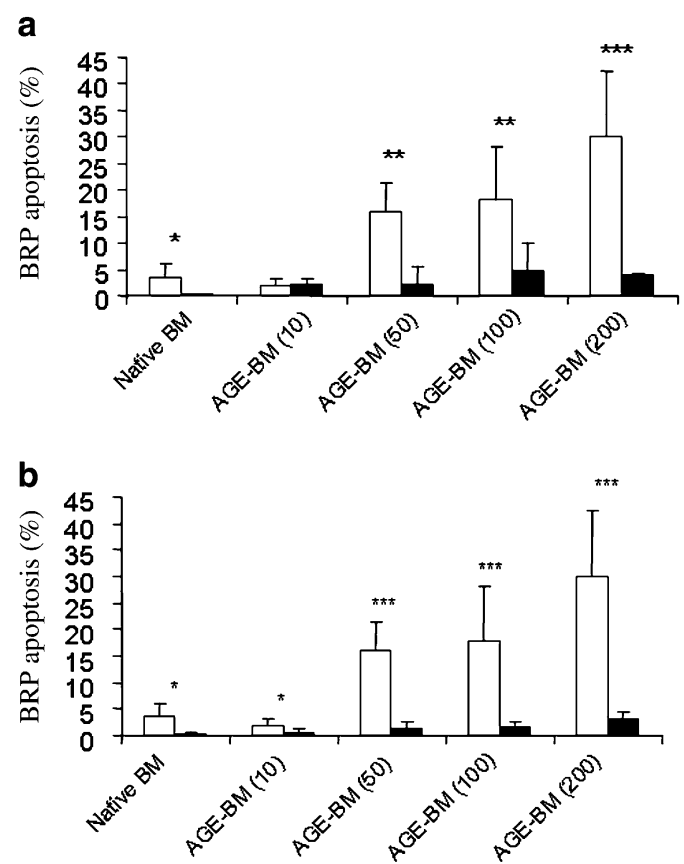

Fig. 7. PDGF rescues retinal pericytes from AGE-modifiedBM-induced apoptotic death. When phase-bright, roundedup pericytes were quantified, there was a clear increase in apoptotic cells when exposed to AGE-modified BM, which was in accordance with the degree of AGE modification (10-100 mmol/l glycoaldehyde) (a, b). * ${ }^{*}<0.05 ; * * p<0.01$; $* * * \quad p<0.001$. When the substrates were supplemented with PDGF-AA peptide (a) or PDGF-BB peptide (b) there was a significant reduction in apoptosis, in many cases, bringing levels down to native BM levels. Open bars: BM not exposed to PDGF; black bars: BM supplemented with PDGF. Error bars represent $\mathrm{SD} \pm$ mean
Using this diabetes-related phenomenon as a basis for the in vitro model system employed, the current investigation demonstrated that AGE modification of a relevant sub-cellular substrate can cause impaired replicative capacity in retinal pericytes during shortterm culture. This reflects an AGE-mediated dysregulation of normal cell growth dynamics and has been previously demonstrated in several other studies using AGE-modified serum proteins [41, 42]. Pericytes show very low mitotic activity in vivo [43] therefore studies of growth dynamics should be taken as a reflection of a generalised dysfunctional response, which is difficult to relate to pericytes as they reside on capillaries in vivo. Of more relevance to retinal capillary dysfunction during diabetes is the finding that relatively prolonged incubation on AGE-modified $\mathrm{BM}$ induces widespread apoptotic death in pericytes.

Soluble AGE are known to induce apoptotic death in retinal pericytes $[11,44]$, a response that may be associated with increased oxidative stress [45] and inhibited by treatment with antioxidants $[44,46]$ or protective growth factors [47]. There may also be an association with AGE-receptor modulation of this response [46]. Many previous studies of AGE-mediated pericyte dysfunction and death have used AGEmodified serum proteins, which may be more meaningful during late-stage diabetic retinopathy when overt breakdown of the blood retinal barrier has occurred. However, pericytes are sequestered within an envelope of BM and reside within the protection of the inner blood retinal barrier. In studies of progressive diabetic retinopathy, AGE modification of the pericyte subcellular matrix may create a more anatomically relevant model system that mimics what would be expected to occur in diabetic vessels over many years.

BM-immobilised AGE may play an important pathogenic role in pericyte dysfunction and/or death, since these cells are reliant on key trophic factors being transported through this matrix from the microvascular endothelium, where they interact with heparan sulphate proteoglycans (HSP), serving to prolong

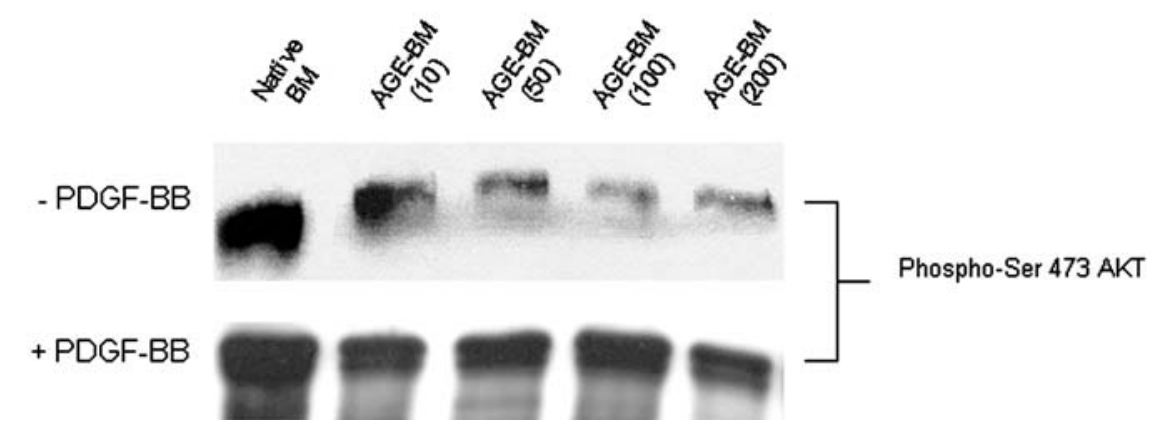

Fig. 8. Supplementation of AGE-modified BM with PDGF-BB restores Akt phosphorylation in retinal pericytes. Retinal pericytes grown on AGE-modified BM for 24 hours showed reduced phosphorylation of Akt, which reduced as AGE modifi-

cation increased. When PDGF-BB was added to the substrate prior to cell seeding, Akt phosphorylation levels were restored to native BM levels 
bioactivity and enhance cell-receptor interactions [48, 49]. Advanced glycation reactions could have a major role in altering physiological properties of vascular BMs such as electrostatic charge, tertiary structure, macromolecule passage and HSP content [20, 50, 51, 52]. It has been demonstrated that AGE modification of matrices can dramatically alter cell viability including vascular cell functions such as angiogenesis [53]. Furthermore, in retinal pericytes seeded onto an endothelium-secreted matrix within a high-glucose environment, appropriate adhesion is prevented, inducing apoptotic death, responses that can be inhibited by co-treatment with the AGE-inhibitor aminoguanidine $[54,55]$.

Supplementation of AGE-modified BM with exogenous PDGF can reverse some of the dysfunctional effects in retinal pericytes. This finding is supported by the fact that PDGF isoforms are established survival factors for pericytes in other non-retinal vascular beds [25]. The role of this growth factor in diabetic retinopathy is also exemplified by the recent report that effective removal of PDGF-BB (as occurs in PDGF-BB null mice) causes depletion of retinal pericytes, an effect which is exacerbated when experimental diabetes is induced in this heterozygous transgenic model $[13,29]$. During diabetes, AGE cross-linking of the BM may serve to reduce effective transmittance of PDGF isoforms to the pericyte, whether they are derived from serum or secreted by endothelium and/or cells of the retinal neuropile. Interestingly, the diabetic milieu may also serve to reduce PDGF (AA and BB isoforms) mRNA expression in the retina [39] and this would further exacerbate a reduced bioavailability of this important pericyte survival factor. It remains possible that an effective depletion of PDGF within vascular BMs occurs during diabetes, and this may predispose pericytes to death via a lack of survival factor stimulation. In addition, the efficacy of many growth factors is known to be significantly reduced by modifying glycation reactions [56]. The up-regulation of PDGF-receptor mRNAs shown in the current study would be consistent with this hypothesis and would reflect a pericyte response to low peptide bioavailability, effected to maintain activation of important prosurvival pathways such as phosphorylation of Akt as a downstream consequence of the dimerisation of PDGF tyrosine kinase receptors [57].

The current investigation provides evidence that AGE modification of BM could play an important role in retinal pericyte survival during diabetes. Rescue from apoptotic death by supplementation of these matrices with PDGF indicates the primary role played by this growth factor in retinal capillary viability, and suggests a mechanism whereby AGE modification could promote progression of diabetic retinopathy. Manipulation of this growth factor and its receptor during diabetes may help prevent aspects of retinopathy.
Acknowledgements. The authors acknowledge support from The Health Research Board (HRB) and The Research and Development Office (NI) (to A.W. Stitt). This work was also partially supported by NIH grant, NIDDK 19971 (to S.R. Thorpe).

\section{References}

1. Hirschi KK, D'Amore PA (1996) Pericytes in the microvasculature. Cardiovasc Res 32:687-698

2. Wu DM, Kawamura H, Sakagami K, Kobayashi M, Puro DG (2003) Cholinergic regulation of pericyte-containing retinal microvessels. Am J Physiol Heart Circ Physiol 284:H2083-H2090

3. Kelley C, D'Amore P, Hechtman HB, Shepro D (1987) Microvascular pericyte contractility in vitro: comparison with other cells of the vascular wall. J Cell Biol 104:483490

4. D'Amore PA, Smith SR (1993) Growth factor effects on cells of the vascular wall: a survey. Growth Factors 8:61-75

5. Garner A (1993) Histopathology of diabetic retinopathy in man. Eye 7:250-253

6. Li W, Yanoff M, Liu X, Ye X (1997) Retinal capillary pericyte apoptosis in early human diabetic retinopathy. Chin Med J (Engl) 110:659-663

7. Podesta F, Romeo G, Liu WH et al. (2000) Bax is increased in the retina of diabetic subjects and is associated with pericyte apoptosis in vivo and in vitro. Am J Pathol 156:1025-1032

8. Kern TS, Tang J, Mizutani M et al. (2000) Response of capillary cell death to aminoguanidine predicts the development of retinopathy: comparison of diabetes and galactosemia. Invest Ophthalmol Vis Sci 41:3972-3978

9. Murata M, Ohta N, Fujisawa S et al. (2002) Selective pericyte degeneration in the retinal capillaries of galactose-fed dogs results from apoptosis linked to aldose reductasecatalyzed galactitol accumulation. J Diabetes Complications 16:363-370

10. Miwa K, Nakamura J, Hamada Y et al. (2003) The role of polyol pathway in glucose-induced apoptosis of cultured retinal pericytes. Diabetes Res Clin Pract 60:1-9

11. Yamagishi S, Amano S, Inagaki Y et al. (2002) Advanced glycation end products-induced apoptosis and overexpression of vascular endothelial growth factor in bovine retinal pericytes. Biochem Biophys Res Commun 290:973-978

12. Stitt AW, Jenkins AJ, Cooper ME (2002) Advanced glycation end products and diabetic complications. Expert Opin Investig Drugs 11:1205-1223

13. Hammes HP (2003) Pathophysiological mechanisms of diabetic angiopathy. J Diabetes Complications 17:16-19

14. Baynes JW, Thorpe SR (2000) Glycoxidation and lipoxidation in atherogenesis. Free Radic Biol Med 28:17081716

15. Stitt AW, Li YM, Gardiner TA, Bucala R, Archer DB, Vlassara H (1997) Advanced glycation end products (AGEs) co-localize with AGE receptors in the retinal vasculature of diabetic and of AGE-infused rats. Am J Pathol 150:523-531

16. Stitt A, Gardiner TA, Alderson NL et al. (2002) The AGE inhibitor pyridoxamine inhibits development of retinopathy in experimental diabetes. Diabetes 51:2826-2832

17. Hammes HP, Alt A, Niwa T et al. (1999) Differential accumulation of advanced glycation end products in the course of diabetic retinopathy. Diabetologia 42:728-736 
18. Frisch SM, Ruoslahti E (1997) Integrins and anoikis. Curr Opin Cell Biol 9:701-706

19. Cardone MH, Roy N, Stennicke HR et al. (1998) Regulation of cell death protease caspase- 9 by phosphorylation. Science 282:1318-1321

20. Bailey AJ (2001) Molecular mechanisms of ageing in connective tissues. Mech Ageing Dev 122:735-755

21. Chakravarthy U, Gardiner TA (1999) Endothelium-derived agents in pericyte function/dysfunction. Prog Retin Eye Res 18:511-527

22. Hart CE, Forstrom JW, Kelly JD et al. (1988) Two classes of PDGF receptor recognize different isoforms of PDGF. Science 240:1529-1531

23. Matsui T, Pierce JH, Fleming TP et al. (1989) Independent expression of human alpha or beta platelet-derived growth factor receptor cDNAs in a naive hematopoietic cell leads to functional coupling with mitogenic and chemotactic signaling pathways. Proc Natl Acad Sci USA 86:83148318

24. Rosenkranz S, Kazlauskas A (1999) Evidence for distinct signaling properties and biological responses induced by the PDGF receptor alpha and beta subtypes. Growth Factors 16:201-216

25. Lindahl P, Johansson BR, Leveen P, Betsholtz C (1997) Pericyte loss and microaneurysm formation in PDGF-Bdeficient mice. Science 277:242-245

26. Bostrom H, Willetts K, Pekny M et al. (1996) PDGF-A signaling is a critical event in lung alveolar myofibroblast development and alveogenesis. Cell 85:863-873

27. Soriano P (1994) Abnormal kidney development and hematological disorders in PDGF beta-receptor mutant mice. Genes Dev 8:1888-1896

28. Hammes HP, Lin J, Renner O et al. (2002) Pericytes and the pathogenesis of diabetic retinopathy. Diabetes 51:31073112

29. Enge M, Bjarnegard M, Gerhardt H et al. (2002) Endothelium-specific platelet-derived growth factor-B ablation mimics diabetic retinopathy. EMBO J 21:4307-4316

30. Chakravarthy U, Stitt AW, McNally J, Bailie JR, Hoey EM, Duprex P (1995) Nitric oxide synthase activity and expression in retinal capillary endothelial cells and pericytes. Curr Eye Res 14:285-294

31. Yamagishi S, Yonekura H, Yamamoto Y et al. (1997) Advanced glycation end products-driven angiogenesis in vitro. Induction of the growth and tube formation of human microvascular endothelial cells through autocrine vascular endothelial growth factor. J Biol Chem 272:8723-8730

32. Nagai R, Matsumoto K, Ling X, Suzuki H, Araki T, Horiuchi S (2000) Glycolaldehyde, a reactive intermediate for advanced glycation end products, plays an important role in the generation of an active ligand for the macrophage scavenger receptor. Diabetes 49:1714-1723

33. Shaw JN, Baynes JW, Thorpe SR (2002) N epsilon-(carboxymethyl)lysine (CML) as a biomarker of oxidative stress in long-lived tissue proteins. Methods Mol Biol 186:129-137

34. Stegemann H, Stalder K (1967) Determination of hydroxyproline. Clin Chim Acta 18:267-273

35. Simpson DA, Feeney S, Boyle C, Stitt AW (2000) Retinal VEGF mRNA measured by SYBR green I fluorescence: a versatile approach to quantitative PCR. Mol Vis 6:178183

36. Diaz G, Setzu MD, Zucca A et al. (1999) Subcellular heterogeneity of mitochondrial membrane potential: relationship with organelle distribution and intercellular contacts in normal, hypoxic and apoptotic cells. J Cell Sci 112:1077-1084
37. Hammes HP, Wellensiek B, Kloting I, Sickel E, Bretzel RG, Brownlee M (1998) The relationship of glycaemic level to advanced glycation end-product (AGE) accumulation and retinal pathology in the spontaneous diabetic hamster. Diabetologia 41:165-170

38. Gardiner TA, Anderson HR, Stitt AW (2003) AGE-inhibition protects against retinal capillary basement membrane thickening during long-term diabetes. J Pathol 201:328333

39. Cox O, Stitt AW, Simpson DA, Gardiner TA (2003) Sources of PDGF expression in murine retina and the effect of short-term diabetes. Mol Vis 10:665-672

40. Degenhardt TP, Alderson NL, Arrington DD et al. (2002) Pyridoxamine inhibits early renal disease and dyslipidemia in the streptozotocin-diabetic rat. Kidney Int 61:939-950

41. Chibber R, Molinatti PA, Wong JS, Mirlees D, Kohner EM (1994) The effect of aminoguanidine and tolrestat on glucose toxicity in bovine retinal capillary pericytes. Diabetes 43:758-763

42. Ruggiero-Lopez D, Rellier N, Lecomte M, Lagarde M, Wiernsperger N (1997) Growth modulation of retinal microvascular cells by early and advanced glycation products. Diabetes Res Clin Pract 34:135-142

43. Sharma NK, Gardiner TA, Archer DB (1985) A morphologic and autoradiographic study of cell death and regeneration in the retinal microvasculature of normal and diabetic rats. Am J Ophthalmol 100:51-60

44. Denis U, Lecomte M, Paget C, Ruggiero D, Wiernsperger N, Lagarde M (2002) Advanced glycation end-products induce apoptosis of bovine retinal pericytes in culture: involvement of diacylglycerol/ceramide production and oxidative stress induction. Free Rad Biol Med 33:236-247

45. Yamagishi S, Okamoto T, Amano S et al. (2002) Palmitateinduced apoptosis of microvascular endothelial cells and pericytes. Mol Med 8:179-184

46. Yamagishi S, Amano S, Inagaki Y, Okamoto T, Takeuchi M, Makita Z (2002) Beraprost sodium, a prostaglandin I2 analogue, protects against advanced gycation end productsinduced injury in cultured retinal pericytes. Mol Med $8: 546-550$

47. Yamagishi S, Inagaki Y, Amano S, Okamoto T, Takeuchi M, Makita Z (2002) Pigment epithelium-derived factor protects cultured retinal pericytes from advanced glycation end product-induced injury through its antioxidative properties. Biochem Biophys Res Commun 296:877-882

48. Iozzo RV, San Antonio JD (2001) Heparan sulfate proteoglycans: heavy hitters in the angiogenesis arena. J Clin Invest 108:349-355

49. Vlodavsky I, Miao HQ, Medalion B, Danagher P, Ron D (1996) Involvement of heparan sulfate and related molecules in sequestration and growth promoting activity of fibroblast growth factor. Cancer Metastasis Rev 15:177186

50. Boyd-White J, Williams JC Jr (1996) Effect of cross-linking on matrix permeability. A model for AGE-modified basement membranes. Diabetes 45:348-353

51. Mott JD, Khalifah RG, Nagase H, Shield CF 3rd, Hudson JK, Hudson BG (1997) Nonenzymatic glycation of type IV collagen and matrix metalloproteinase susceptibility. Kidney Int 52:1302-1312

52. Sensi M, Tanzi P, Bruno MR et al. (1989) Nonenzymic glycation of isolated human glomerular basement membrane changes its physicochemical characteristics and binding properties. Nephron 52:222-226

53. Kuzuya M, Satake S, Ai S et al. (1998) Inhibition of angiogenesis on glycated collagen lattices. Diabetologia 41:491499 
54. Beltramo E, Pomero F, Allione A, D’Alu F, Ponte E, Porta M (2002) Pericyte adhesion is impaired on extracellular matrix produced by endothelial cells in high hexose concentrations. Diabetologia 45:416-419

55. Beltramo E, Buttiglieri S, Pomero F et al. (2003) A study of capillary pericyte viability on extracellular matrix produced by endothelial cells in high glucose. Diabetologia 46:409-415
56. Giardino I, Edelstein D, Brownlee M (1994) Nonenzymatic glycosylation in vitro and in bovine endothelial cells alters basic fibroblast growth factor activity. A model for intracellular glycosylation in diabetes. J Clin Invest 94:110-117

57. Taylor CC (2000) Platelet-derived growth factor activates porcine thecal cell phosphatidylinositol-3-kinase-Akt/PKB and ras-extracellular signal-regulated kinase-1/2 kinase signaling pathways via the platelet-derived growth factor-beta receptor. Endocrinology 141:1545-1553 